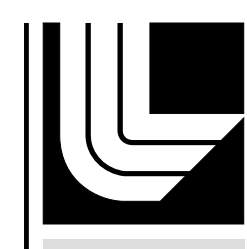

LA W RENCE LIVERM ORE NATIONAL LABORATORY

\title{
Characteristics of Undoped and Europium-doped Srl2 Scintillator Detectors
}

B. W. Sturm, N. J. Cherepy, O. B. Drury, P. A. Thelin, S. E. Fisher, S. A. Payne, A. Burger, L. Boatner, K. Shah

November 15, 2011

IEEE Nuclear Science Symposium Valencia, Spain October 24, 2011 through October 28, 2011 
This document was prepared as an account of work sponsored by an agency of the United States government. Neither the United States government nor Lawrence Livermore National Security, LLC, nor any of their employees makes any warranty, expressed or implied, or assumes any legal liability or responsibility for the accuracy, completeness, or usefulness of any information, apparatus, product, or process disclosed, or represents that its use would not infringe privately owned rights. Reference herein to any specific commercial product, process, or service by trade name, trademark, manufacturer, or otherwise does not necessarily constitute or imply its endorsement, recommendation, or favoring by the United States government or Lawrence Livermore National Security, LLC. The views and opinions of authors expressed herein do not necessarily state or reflect those of the United States government or Lawrence Livermore National Security, LLC, and shall not be used for advertising or product endorsement purposes. 


\title{
Characteristics of Undoped and Europium- doped $\mathrm{SrI}_{2}$ Scintillator Detectors
}

\author{
Benjamin W. Sturm, Member, IEEE, Nerine J. Cherepy, Member, IEEE, Owen B. Drury, Peter A. Thelin, Scott E. \\ Fisher, Sean P. O'Neal, Stephen A. Payne, Member, IEEE, Arnold Burger, Member, IEEE, Lynn A. Boatner, \\ Member, IEEE, Joanne O. Ramey, Kanai S. Shah, Member, IEEE, Rastgo Hawrami, Member, IEEE
}

\begin{abstract}
High energy resolution gamma-ray detectors that can be formed into relatively large sizes while operating at room temperature offer many advantages for national security applications. We are working toward that goal through the development of $\mathrm{SrI}_{2}(\mathrm{Eu})$ scintillator detectors, which have already achieved $<3.0 \%$ energy resolution at $662 \mathrm{keV}$ with volumes $>10 \mathrm{~cm}^{3}$. In this study, we have tested pure, undoped $\mathrm{SrI}_{2}$ to gain a better understanding of the scintillation properties and spectroscopic performance achievable without activation. An undoped crystal grown from $99.999 \%$ pure $\mathrm{SrI}_{2}$ pellets was tested for its spectroscopic performance, its light yield, and uniformity of scintillation light collection as a function of gammaray interaction position relative to the crystal growth direction. We measured an energy resolution of $5.3 \%$ at $662 \mathrm{keV}$, and the light collection non-uniformity varied by only $0.72 \%$ over the length of the crystal. Measurements of both a $3 \% \mathrm{Eu}$-doped and the undoped $\mathrm{SrI}_{2}$ detector were carried out in the SLYNCI facility to determine their light yield non-proportionality, which will be reported. The surprisingly good scintillation properties of the pure $\mathrm{SrI}_{2}$ crystal suggests that with high-purity feedstock, further reduction of the Eu concentration can be made to grow larger crystals while not adversely impacting the spectroscopic performance.
\end{abstract}

\section{INTRODUCTION}

$\mathrm{SrI}_{2}(\mathrm{Eu})$ crystals are being grown, polished, and packaged $S_{\text {with high yield and consistently good spectroscopic }}$ performance. We are now able to consistently achieve $\sim 3 \%$ FWHM energy resolution at $662 \mathrm{keV}$ with 1 cubic inch crystals, as was previously reported [1,2]. Two key material parameters that affect $\mathrm{SrI}_{2}(\mathrm{Eu})$ detector performance is the purity of the feedstock and the Eu doping concentration. Both of these parameters affect the light yield and light collection uniformity and, thereby, the achievable energy resolution.

In our earlier work, we measured an energy resolution of $6.7 \%$ for an undoped $\mathrm{SrI}_{2}$ crystal grown from zone-refined feedstock at Fisk University [3]. In the work reported here, a

Manuscript received November 15, 2011. This work was supported by the Department of Homeland Security Domestic Nuclear Detection Office and was performed under the auspices of the U.S. Department of Energy by Lawrence Livermore National Laboratory under Contract DE-AC5207NA27344. LLNL-PROC-462782.

B. W. Sturm, N. J. Cherepy, O. B. Drury, P. A. Thelin, S. E. Fisher, A. F. Magyar, and S. A. Payne are with Lawrence Livermore National Laboratory, Livermore, CA 94550 USA. (corresponding author e-mail: sturm1@ @lnl.gov). A. Burger is with Fisk University, Nashville, TN 37201 USA.

L. A. Boatner and J. O. Ramey are with Oak Ridge National Laboratory, Oak Ridge, TN 37830 USA.

K. S. Shah and R. Hawrami are with Radiation Monitoring Devices, Watertown, MA 02472 USA. crystal was grown from $99.999 \%$ pure $\mathrm{SrI}_{2}$ (Sigma Aldrich) by Oak Ridge National Laboratory (ORNL), cut, polished and packaged in a hermetic enclosure at Lawrence Livermore National Laboratory. Its performance is compared with a 3\% Eu-doped $\mathrm{SrI}_{2}$ crystal grown by RMD, Inc. that was polished and encapsulated at Lawrence Livermore National Laboratory. A comparison study of this effect allows us to decouple the effects associated with the europium activator, giving us better insight into the properties of the $\mathrm{SrI}_{2}$ host material.

\section{METHODS}

\section{A. Scintillator Emission and Decay Measurements}

One method by which to understand the physics of light generation in scintillator detectors is through measurement of the emission spectra. These measurements were acquired using a beta source to excite the scintillator. The light was then collected with a spectrograph coupled to a thermoelectrically cooled camera (perhaps Nerine can expand on this?). Emission spectra were recorded for the very pure ORNL undoped crystal, the Fisk undoped crystal, and the RMD 3\% Eu-doped $\mathrm{SrI}_{2}$ crystal. In addition, an absorption spectrum was recorded for the ORNL crystal.

Scintillator decay times were also measured for the undoped and 3\% Eu-doped crystals using a Bridgeport Emorpho data acquisition system. With this readout system, scintillator pulse traces were recorded and those pulses which constituted a full-energy deposition were selected. 10 traces from the selected pulses were averaged to give a normalized response. $\mathrm{A}{ }^{137} \mathrm{Cs}$ source was used for this measurement.

\section{B. Spectroscopic Characteristics for Packaged and Unpackaged Crystals.}

We measured the spectroscopic performance of two packaged detectors, an undoped ORNL crystal $8.0 \mathrm{~cm}^{3}$ in volume, and a $3 \%$ Eu-doped RMD crystal $10.4 \mathrm{~cm}^{3}$ in volume. These crystals were packaged using our standard technique [2]. Spectra were recorded using a ${ }^{137} \mathrm{Cs}$ source and an effective light yield was determined by normalizing the signal to a reference $\mathrm{NaI}(\mathrm{Tl})$ detector.

We also acquired measurements of the light collection nonuniformity as described previously [2]. In this measurement, a collimated ${ }^{137} \mathrm{Cs}$ source is used to measure the scintillator response as a function of gamma-ray interaction depth (i.e. distance from the PMT window). From this, we can understand the light collection nonuniformity as a function of interaction depth as well as the spectroscopic characteristics 
as a function of depth. We used unpackaged cylindrical crystals for these measurements, because we have found that the effects of light collection nonuniformity are exacerbated in this scenario (versus the packaged crystal). Both crystals were wrapped with $\sim 8$ layers of Teflon tape, and coupled to a PMT with a thin layer of mineral oil.

\section{SLYNCI Measurements of the Light Yield Nonproportionality}

In this study, we also acquired measurements of the light yield nonproportionality. Such measurements are important to quantify, since the allowable energy resolution of a scintillator detector is partially determined by its light yield nonproportionality. In addition, these measurements also give us further understanding of the physics of light generation. These measurements were carried out at the Scintillator Light Yield Nonproportionality Characterization Instrument (SLYNCI) $[4,5]$. It is important to point out that the SLYNCI instrument measures the relative light yield as a function of electron response (e.g. energy imparted to the Compton scatter electron) as opposed to the gamma-ray response (e.g. energy of the incident gamma-ray). This is considered to be a more fundamental measure of the true nonproportionality behavior of the scintillator. SLYNCI measurements of the packaged ORNL undoped $\mathrm{SrI}_{2}$ crystal as well as the packaged RMD 3\% Eu-doped $\mathrm{SrI}_{2}$ crystal were recorded.

\section{D. $\mathrm{SrI}_{2}$ index of refraction measurements}

The final part of this study involved measurements of the refractive index of $\mathrm{SrI}_{2}$. The refractive index is a key material property to quantify, since this can help us to better model the physics of light transport in the material. To date, the only index of refraction number that has been reported was determined through quantum calculations, resulting in a computed index value of 2.05-2.07 at $435 \mathrm{~nm}$ [6]. We decided to do experimental measurements of this important parameter using the method of minimum deviation (find reference). In this method, the material of interest is shaped into a prism, with an apex angle of $\alpha$. A collimated and coherent beam of light is then directed toward the prism. We then scan the beam from $0^{\circ}$ to $90^{\circ}$ incident angle and observe the angle by which the scattered beam is diverted from the incident beam. The minimum angle at which the scattered beam is diverted is known as the minimum deviation angle $D$. The index of refraction value $n$ can then be determined based on the following relationship

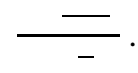

Measurements of the index of refraction were carried out in an $\mathrm{N}_{2}$-filled dry box. For ease of use, two laser pointers were used in this measurement, one which emits in the red $(\lambda=650$ $\mathrm{nm})$ and the other which emits in the green $(\lambda=532 \mathrm{~nm})$. A $5 \%$ Eu-doped $\mathrm{SrI}_{2}$ crystal was shaped into a prism with $\alpha=40^{\circ}$. The crystal was mounted on a rotation stage to allow us to accurately measure the minimum deviation angle, and thus the index of refraction.

\section{RESULTS AND DISCUSSION}

\section{A. Results for Scintillator Emission, Absorption, and Decay Time Measurements}

The results of the radioluminescence emission spectra for all the crystals measured are shown in Fig. 1. Fig. 1(a) displays the emission and absorption spectra for the undoped ORNL detector, and Fig 1(b) shows just the emission spectra for all the crystals. The emission spectrum in Fig. 1(a) consists of a broad continuum, where the shoulder region of the continuum, centered at about 400nm, is believed to be caused by emission due to self-trapped excitons (STEs), whereas the longer wavelength emission is thought to be from impurity or defectmediated recombination. The STE emission is considered to be an ideal mechanism of deexcitation in the undoped crystal, since its emission is well-matched to bialkali PMTs, and furthermore for the doped crystal, the energy of STE emission is high enough that the light can be absorbed and reemitted by the $\mathrm{Eu}^{2+}$ sites. From the absorption spectrum in Fig. 1(a), we estimated the $\mathrm{SrI}_{2}$ bandgap to be $\sim 4.4 \mathrm{eV}$.

In Fig. 1(b) we observe that the doped spectrum (in blue) has a prominent emission centered at $435 \mathrm{~nm}$, which is from the $\mathrm{Eu}^{2+}$ activator. The doped spectrum also exhibits a long-waved emission, like the undoped spectra, indicating that all samples have emission from impurities and/or defects. In Fig. 1(b), we also found that the relative fraction of the long-wave emission, to that of the STE emission, reduced for the crystal grown from the highly purified feedstock. From this observation, we can infer that with higher purity $\mathrm{SrI}_{2}$ feedstock, we may be able to decrease the Eu doping concentration while not sacrificing performance in terms of photoelectron yield. This is important, because the detrimental effects of light trapping will be less severe when the Eu doping concentration is reduced, and thus allowing us to scale the crystal to larger sizes.

Results of the decay time measurements are shown in Fig. 2. It was found that both sets of traces were well matched to a double exponential decay function. The undoped sample had decay time constants of $\tau_{1}=0.106$ us and $\tau_{2}=0.511$ us. The doped sample had decay time constants of $\tau_{1}=0.123$ us and $\tau_{2}$ $=3.488$ us. The fact that the undoped sample exhibits a double exponential decay agrees with the notion that there are more than one decay pathways for the undoped crystal. The fast component for both detectors more or less agrees with each other, and it is thought that this component is associated with the broad band at higher wavelengths. The slower component for the doped sample is a result of $\mathrm{Eu}^{2+}$ emission. Although the intrinsic decay time for $\mathrm{Eu}^{2+}$ is $1.2 \mu \mathrm{s}$, we measured a longer decay time due to absorption and reemission of the $\mathrm{Eu}^{2+}$. This effect has been reported previously [7].

\section{B. Results from Spectroscopic and Light-collection Nonuniformity Measurements}

In Fig. 3, a comparison of the $662 \mathrm{keV}$ spectra for the two detectors is displayed. We measured energy resolutions of $5.28 \%$ FWHM for the undoped sample and 2.85\% FWHM for the doped sample. The surprisingly good energy resolution for the undoped sample (e.g. better than for $\mathrm{NaI}(\mathrm{Tl})$ ) is a 
testament to the intrinsically exceptional qualities of $\mathrm{SrI}_{2}$ for gamma-ray detection and spectroscopy. Based on the relative positions of the photopeaks, we estimated the effective light yield to be 22,600 photons/MeV and 82,400 photons/MeV for the undoped and doped detectors, respectively. It is important to point out that we did not correct for the PMT quantum efficiency in these estimates, so the true absolute light yield for the undoped sample should in fact be higher.

The results from the collimation experiment are shown in Fig. 4. Fig. 4(a) displays the spectra recorded for each slice of the undoped sample, and Fig. 4(b) corresponds to the doped sample. The total amount that the photopeak shifts between events that originate at the top of the crystal (pos1) versus events that originate at the bottom (pos3) is dependent on the light collection nonuniformity. It has been reported that for crystals exhibiting severe light collection nonuniformity, upwards of $10 \%$ photopeak shift can be observed [2]. For the undoped crystal, we found that the total photopeak shift was just $0.72 \%$, whereas for the doped crystal the total photopeak shift was $2.13 \%$. The fact that the undoped sample exhibits a much smaller peak shift is not surprising, because the effect of light collection non-uniformity is associated with optical absorption and reemission of the $\mathrm{Eu}^{2+}$.

Another important observation that we can infer from Fig. 4 is the fact that there was little variation of resolution for the undoped sample, in contrast to the doped sample. This agrees with a model developed previously in which degradation in energy resolution as a function of scan depth is associated with the effects of absorption and reemission (or light trapping) of the $\mathrm{Eu}^{2+}$ [1]. The light trapping effect will cause greater dispersion in the photon pathlengths for events that originate closer to the PMT versus those events that originate further away from the PMT. This dispersion will result in degraded energy resolution. The fact that we see this effect for the doped sample in Fig. 4(b), but not for the undoped sample in Fig. 4(a), helps to validate our model.

\section{SLYNCI Results for the Undoped and 3\% Doped Packaged Detectors}

Data from the SLYNCI measurements is shown in Fig. 5. Here we observe that the relative light yields for the two crystals match well for electron energies $>40 \mathrm{keV}$, but then diverge in the lower energy region. The trends observed here are consistent with a previously reported model of nonproportionality in scintillator detectors [8]. In this model, the rising portion of the curve, from higher energy to lower energy, is associated with the Onsager mechanism, which can be summarized as the competition between exciton formation and exciton-exciton annihilation. This mechanism is considered to be dependent on the intrinsic parameters of the host material, and therefore we would expect the two curves to coalign as they do. In the model, the falling portion of the curve however is associated with the Birks mechanism, which is related to carrier and exciton mobilities and the activator and trap concentrations, and therefore the doping and purity of the material. Therefore, we would anticipate that these two curves would differ between the doped and undoped samples. Luckily, the falling portion of the curve is less severe for the doped sample, resulting in very good light yield proportionality, and thus intrinsically very good energy resolution.

\section{Results from Index of Refraction Measurements}

Experimental results for the index of refraction measurements are given in Table 1. Like most materials, the index of refraction is a function of the wavelength. For the green laser source $(\lambda=532 \mathrm{~nm})$, the index of refraction $n=$ $1.89 \pm 0.02$. For the red laser source $(\lambda=650 \mathrm{~nm}), n=1.87 \pm$ 0.02 . The experimental results are in fact smaller than the results determined through computational calculations. Although the cause of this discrepancy is unknown, we would put more faith in the experimental results. In order to validate these results, ellipsometry measurements are also planned for the $\mathrm{SrI}_{2}$ crystals, which has been shown to be a very accurate method for determining index of refraction. We plan to report these results in the future.

\section{CONCLUSIONS}

One of the main goals in this study was to better understand the characteristics of undoped $\mathrm{SrI}_{2}$ grown from $99.999 \%$ pure feedstock. We achieved this by measuring a variety of its scintillator properties, and comparing them to a 3\% Eu-doped crystal. The emission spectra were recorded and we determined that there were two modes of deexcitation in the undoped sample. We found that the optimal mode of deexcitation, from STE emission, was more pronounced for the highly purified material. The decay time measurements showed that both the undoped and doped samples exhibited a double exponential decay, and the fast component for both samples was very similar. The energy resolution for the undoped and doped packaged detectors was determined and we measured $5.28 \%$ and $2.85 \%$ FWHM at $662 \mathrm{keV}$, respectively. Collimation measurements showed that the total photopeak shift for the undoped sample was just $0.72 \%$, which helped to confirm that light collection nonuniformity is a result of light absorption and reemission at the $\mathrm{Eu}^{2+}$ sites. Measurements of the light yield nonproportionality showed that the undoped sample was less proportional than the doped sample. Finally, we measured the index of refraction of a 5\% Eu doped $\mathrm{SrI}_{2}$ crystal and determined it to be $1.87-1.89$ for the wavelengths examined. 


\section{FIGURES}
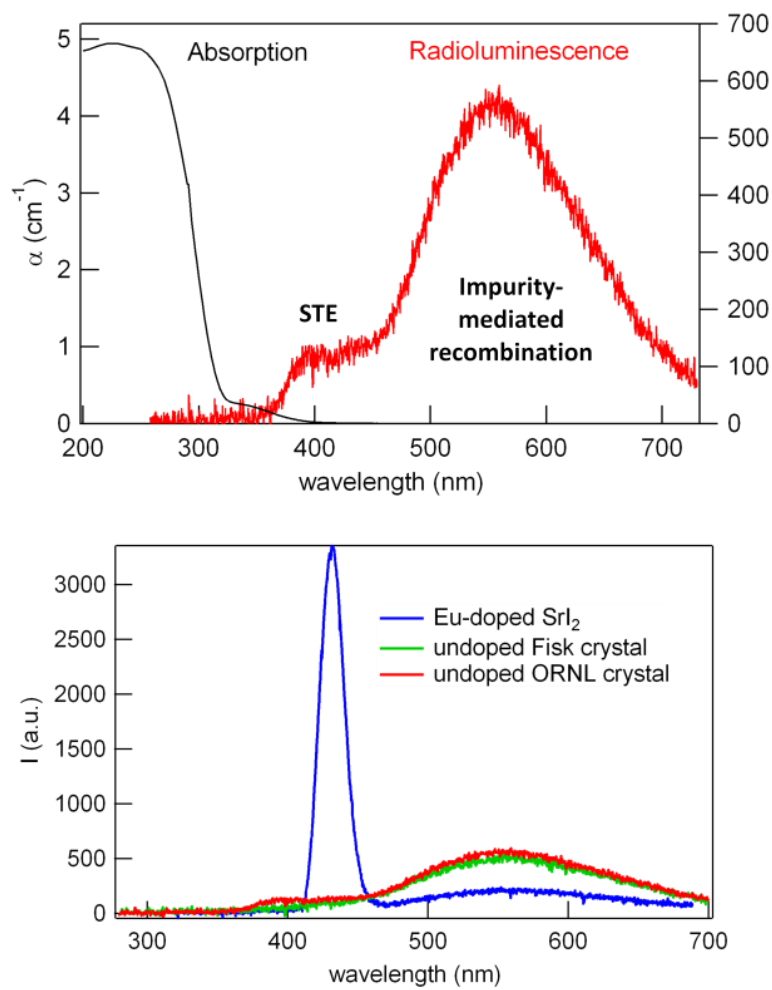

Fig. 1. (a) Measurements of the absorption and emissions spectra for the undoped $99.999 \%$ pure $\mathrm{SrI}_{2}$ ORNL crystal and (b) a comparison of the emission spectra for two undoped crystals and a 3\% Eu-doped crystal.

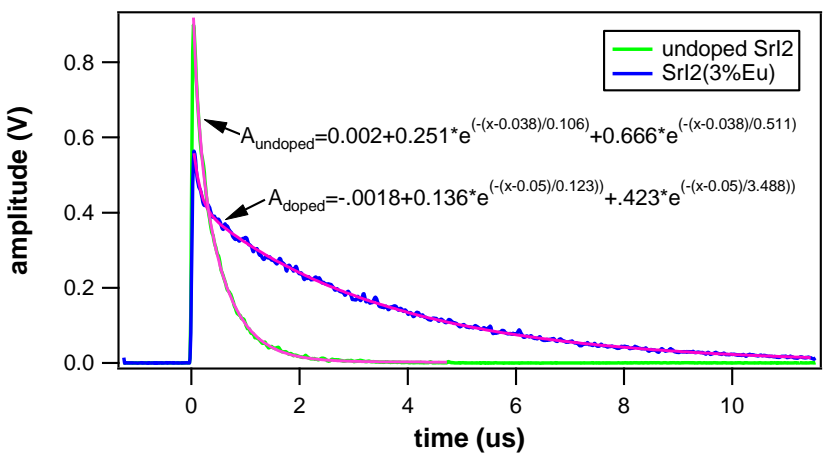

Fig. 2. Scintillator decay traces for the packaged undoped and 3\% Eu-doped detectors. The traces correspond to full energy depositions using a $662 \mathrm{keV}$ source, and were both fit with a double exponential decay function.

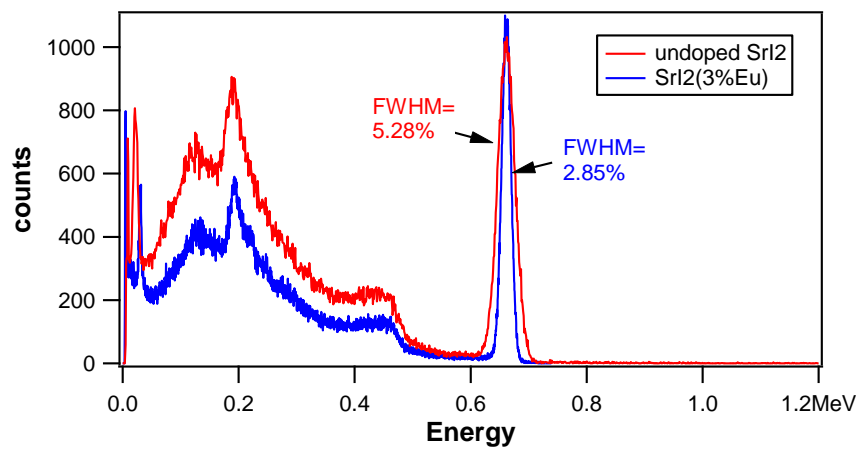

Fig. $3 .{ }^{137} \mathrm{Cs}$ pulse-height spectra for the packaged undoped and 3\% Eu-doped $\mathrm{SrI}_{2}$ detectors. The detector sizes were $8.0 \mathrm{~cm}^{3}$ (undoped) and $10.4 \mathrm{~cm}^{3}$ (doped).
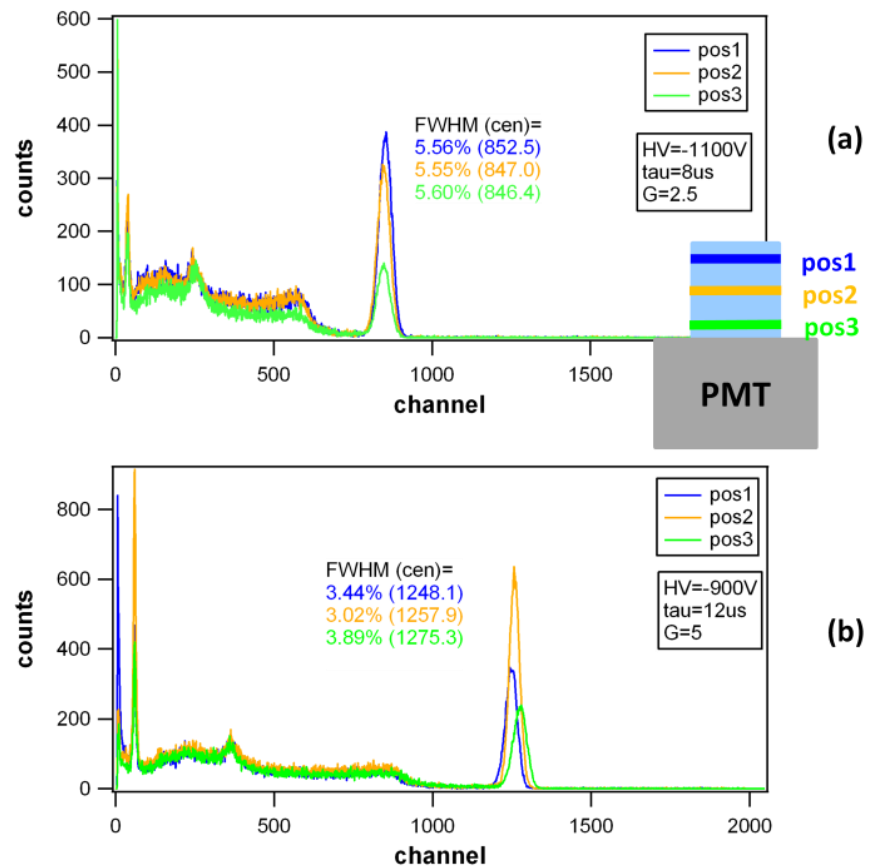

(b)

Fig. 4. Measurements acquired using a collimated ${ }^{137} \mathrm{Cs}$ source, where (a) is the undoped $\mathrm{SrI}_{2}$ and (b) is the $3 \%$ Eu doped crystal. The undoped sample shows a total photopeak variation of $0.72 \%$, whereas for the doped sample, the variation is $2.13 \%$. This observation can be associated with the effect of light trapping in the $\mathrm{Eu}^{2+}$, which is not a factor in the undoped sample.

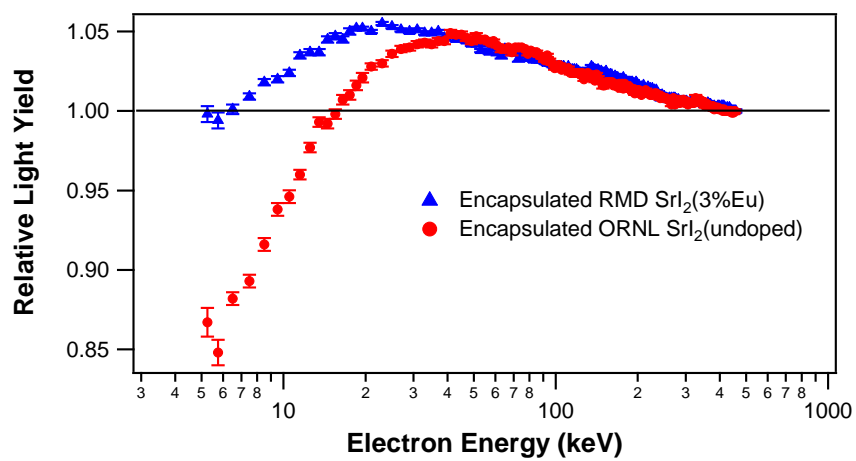

Fig. 5. Light yield nonproportionality data measured at the SLYNCI facility for the undoped and $3 \%$ Eu doped $\mathrm{SrI}_{2}$ crystals. The data indicates that the addition of $\mathrm{Eu}^{2+}$ results in a more proportional scintillation response.

Table 1. Table summarizing the index of refraction measurement results where $\mathrm{D}$ is the minimum deviation angle, $\mathrm{n}$ is the index of refraction, and $\sigma_{\mathrm{n}}$ is the uncertainty.

\begin{tabular}{|l|c|c|c|c|}
\hline \multicolumn{5}{|c|}{ SrI2(5\% Eu) index of refraction data } \\
\hline color & $\begin{array}{c}\text { wavelength } \\
\text { (nm) }\end{array}$ & $\begin{array}{c}\mathbf{D} \\
\text { (degrees) }\end{array}$ & $\mathbf{n}$ & $\boldsymbol{\sigma}_{\mathbf{n}}$ \\
\hline green & 532 & 40.5 & 1.89 & 0.02 \\
\hline red & 650 & 39.33 & 1.87 & 0.02 \\
\hline
\end{tabular}

\section{ACKNOWLEDGEMENTS}

We are especially grateful to Sean Dingman, Sigma Aldrich, for providing highly purified $\mathrm{SrI}_{2}$ feedstock materials.

\section{REFERENCES} Fisher, A. F. Magyar, S. A. Payne, A. Burger, L. A. Boatner, J. O. 
Ramey, K. S. Shah, and R. Hawrami, "Evaluation of Large Volume SrI2(Eu) Scintillator Detectors," IEEE Nuclear Science Symposium, Conference Record, 2010.

[2] B. W. Sturm, N. J. Cherepy, O. B. Drury, P. A. Thelin, S. E. Fisher, S. A. Payne, A. Burger, L. A. Boatner, J. O. Ramey, K. S. Shah, and R. Hawrami, "Effects of packaging SrI2(Eu) scintillator crystals," Nuclear Instruments and Methods in Physics Research Section A: Accelerators, Spectrometers, Detectors and Associated Equipment Symposium on Radiation Measurements and Applications (SORMA) XII 2010, vol. 652, pp. 242-246, 2011/10/1/ 2011.

[3] N. J. Cherepy, S. A. Payne, S. J. Asztalos, G. Hull, J. D. Kuntz, T. Niedermayr, S. Pimputkar, J. J. Roberts, R. D. Sanner, T. M. Tillotson, E. van Loef, C. M. Wilson, K. S. Shah, U. N. Roy, R. Hawrami, A. Burger, L. A. Boatner, C. Woon-Seng, and W. W. Moses, "Scintillators with potential to supersede lanthanum bromide," IEEE Transactions on Nuclear Science, vol. 56, pp. 873-880, June 20092009.

[4] J. D. Valentine and B. D. Rooney, "Design of a Compton Spectrometer Experiment for Studying Scintillator Nonlinearity and Intrinsic Energy Resolution," Nuclear Instruments \& Methods in Physics Research Section a-Accelerators Spectrometers Detectors and Associated Equipment, vol. 353, pp. 37-40, Dec 1994.

[5] W. S. Choong, K. M. Vetter, W. W. Moses, G. Hull, S. A. Payne, N. J. Cherepy, and J. D. Valentine, "Design of a facility for measuring scintillator non-proportionality," Ieee Transactions on Nuclear Science, vol. 55, pp. 1753-1758, Jun 2008.

[6] D. J. Singh, "Near optical isotropy in noncubic $\operatorname{SrI}(2)$ : Density functional calculations," Applied Physics Letters, vol. 92, May 19 2008

[7] J. Glodo, E. V. van Loef, N. J. Cherepy, S. A. Payne, and K. S. Shah, "Concentration Effects in Eu Doped SrI2," Nuclear Science, IEEE Transactions on, vol. 57, pp. 1228-1232, June 20102010.

[8] S. A. Payne, N. J. Cherepy, G. Hull, J. D. Valentine, W. W. Moses, and W. S. Choong, "Nonproportionality of Scintillator Detectors: Theory and Experiment," Ieee Transactions on Nuclear Science, vol. 56, pp. 2506-2512, Aug 2009. 